\title{
LA Filosofia DEL RifiUTO NE LA SOLITUDINE DEL SATIRO DI ENNIO FLAIANO
}

\author{
JESSY CARTON
}

\author{
"Voi siete il sale della terra, \\ ma se il sale diventa sciapo, \\ con che lo saleremo?" (Mat, V-13)
}

\begin{abstract}
Riassunto: In questo articolo si esamina la presunta 'esclusione' di Ennio Flaiano dal campo letterario del secondo Novecento tramite l'analisi della Solitudine del satiro, opera uscita postuma nel 1973. Nell'atteggiarsi a scrittore escluso e restio, Flaiano crea una distanza da cui criticare gli ideali culturali, politici e sociali della società del benessere.
\end{abstract}

Le Opere scelte di Ennio Flaiano (1910-1972), uscite nel 2010 presso Adelphi in occasione del centenario della nascita, riportano all'attenzione uno scrittore 'irregolare' nel panorama della letteratura novecentesca. Anna Longoni, curatrice del volume ${ }^{1}$, ha deciso di includervi tutte le opere narrative edite in vita, recuperando soltanto due scritti postumi ${ }^{2}$, vale a dire quanto "si rivela indispensabile per completare la figura di Flaiano" ("Nota alla presente edizione", LXIII). Benché questa selezione sia comprensibile e pure necessaria data l'enorme quantità di edizioni postume, essa non sembra sufficientemente giustificata. Colpisce in particolare l'esclusione de La solitudine del satiro $(1973)^{3}$, prima opera postuma che fu progettata dall'autore prima della morte ${ }^{4}$ per dare seguito alla linea già iniziata con Diario notturno nel 1956 e continuata con Le ombre bianche nel 1972 (Longoni, "Note ai testi", 1313).

Questa terza raccolta di articoli, apparsi tra il 1956 e il 1972 su Il Mondo, Corriere della Sera e altri quotidiani e periodici, è un collage tipi-

1 La Longoni è anche co-curatrice dei due volumi editi nella serie dei Classici Bompiani, Opere. Scritti postumi (1988) e Opere 1947-1972 (1990).

2 La valigia delle Indie e Diario degli errori.

3 La prima edizione è stata curata da Giulio Cattaneo e Sergio Pautasso. Il presente articolo si riferisce invece all'edizione di Adelphi del 1996, riproduzione di quella allestita da Maria Corti e Anna Longoni per i Classici Bompiani secondo "il criterio della completezza" (Longoni, "Note ai testi" 1313) e indicata con la sigla SS.

4 In un'intervista con Gianni Rosati del 1972 l'autore accenna al progetto (Rosati, 1208). 
camente flaianeo di racconti, pensieri e frammenti di qualità non inferiore alle due pubblicate in vita5. Al di là dell'interesse letterario, la raccolta costituisce inoltre un campione interessante per studiare il concetto di 'irregolarità, termine a tutt'oggi prediletto dalla critica e dalla storiografia per descrivere la posizione dello scrittore pescarese. Pampaloni, per esempio, ha parlato di un "irregolare coerente" (14), la Giammattei di un "letterato ai margini della letteratura" (120), e anche la critica più recente preferisce etichette oscillanti tra "irregolare", "marginale" (Celenza, 30), "voice from the margins" (Trubiano), "marziano" (Schembri) e "anti-italiano" (Ritrovato; D’Angelo). La classificazione è comunemente basata sull'attività pluridimensionale di Flaiano (sceneggiatore, giornalista, scrittore, drammaturgo, critico cinematografico e teatrale) e sulla difficile collocazione delle sue opere in correnti o generi letterari specifici. Tuttavia bisogna chiarire che la posizione ai margini del canone letterario deriva anche in buona parte dal modo in cui Flaiano si presenta nei suoi testi: come uno scrittore solitario che rifiuta di trovarsi al centro di una società e di una letteratura che disapprova. Il presente articolo intende dimostrare come quest'autore, che dice di scrivere "per non essere incluso" (Roda, 18), abbia costruito la propria 'esclusione' per procurarsi una distanza da cui poter criticare le evoluzioni socio-politiche del dopoguerra nonché il modus vivendi e operandi dei letterati nell'Italia del benessere. Gli articoli raccolti ne La solitudine del satiro offrono il campione più adatto per studiare questo concetto perché essi sono in buona parte autobiografici, al contrario de Le ombre bianche, e coprono un arco di tempo sensibilmente più lungo rispetto a quelli del Diario notturno, il che permette di analizzare alcune costanti tematiche collegate al tema dell'esclusione.

È importante notare che la solitudine del satiro è in fondo una solitudine apparente, creata da uno dei protagonisti della vita intellettuale romana tra gli anni Cinquanta e Settanta, habitué del Caffe Rosati e collaboratore fisso di Fellini. In alcuni articoli della raccolta, Flaiano descrive incontri con scrittori e registi come Pasolini, Moravia, Arbasino, Carlo Levi, Antonioni e De Sica, mostrando di conoscerli bene; altrove racconta che sta lavorando con Fellini a "rispolverare una nostra vecchia idea per un film, quella del giovane provinciale che viene a Roma a fare il giornalista" (SS, 235), cioè La dolce vita; o rende conto di attività culturali, quali il

5 Infatti il libro fu accolto positivamente dalla critica, come nota Anna Longoni ("Fortuna critica", 1479). Secondo Marabini è perfino "molto probabile che $L a$ solitudine del satiro resti il libro migliore [di Flaiano], o almeno quello che meglio lo esprime" (III). 
festival del cinema a Venezia e la presentazione del romanzo di Giorgio Bassani nel "sotterraneo della libreria Einaudi pieno zeppo di critici e di scrittori” (SS, 268). Gli esempi qui riportati evidenziano come lo scrittore abruzzese facesse parte del milieu intellettuale dell'epoca, pur cercando di criticare o parodiare le pratiche allora in voga.

La posizione particolare di Flaiano può spiegarsi mediante il concetto di paratopie, concepito da Maingueneau per descrivere la relazione paradossale tra inclusione ed esclusione di uno scrittore in un dato contesto letterario (Dictionnaire, 420). Ai fini del nostro discorso è particolarmente interessante l'osservazione dello studioso francese su quegli scrittori che pretendono di non appartenere a nessuna corrente o categoria:

Le discours littéraire inclut nombre d'écrivains qui prétendent œuvrer hors de toute appartenance; mais c'est justement une des caractéristiques de ce type de discours que de susciter une telle prétention [...]. Les "solitaires" peuvent sans doute s'éloigner des villes, mais non sortir de l'espace qui leur confere leur statut et sur lequel ils proposent leurs actes symboliques. (Le discours littéraire, 54)

In altre parole, anche quando lo smentisce uno scrittore è sempre incluso in un dato ambiente storico-culturale e in una specifica tradizione letteraria. Partendo da questa considerazione, il presente articolo analizzerà in quale modo e per quale motivo Flaiano si presentasse come escluso dalla società e dal campo letterario del dopoguerra ne La solitudine del satiro.

Già il titolo "arguto e malinconico" (Cattaneo, 143) ideato da Flaiano suggerisce un'idea di esclusione, non soltanto per l'uso della parola solitudine, ma anche per il riferimento al satiro. È probabile che quest'ultimo non corrisponda in primis al significato corrente della parola, che si richiama a una creazione mitologica tra umana e caprina, "prototipo dei demoni della fecondità" (Brilli, 10), che racchiude comunque un'idea di anomalia e di diversità non gradite dalla società. Tuttavia il titolo sembra piuttosto alludere al valore che il vocabolo ha assunto nel corso dei secoli quale "simbolo stesso della libertà verbale, tesa al rovesciamento di valori costituiti o all'invettiva scoronante contro i potenti” $(S S, 11)$, avvicinandosi così al noto significato di satirico, benché di origine etimologica diversa ${ }^{6}$. In ogni caso, il titolo nel suo insieme evoca un'idea di isolamento che è intimamente collegata a un sentimento di diversità.

6 "Il fascino di questa associazione che è il satirico-satiro conserva tutto il proprio alone seducente." (Brilli, 11) Anche Paolo Milano suppone che Flaiano alluda "oltre che all' Orazio satiro' di Dante, al satiro dei boschi, quello dal piede caprino, e magari all'insidiatore di donne come lo si chiama in cronaca nera” (148). 
Questa idea è inoltre esplicitata in numerosi articoli della raccolta in cui l'autore insiste sulla propria diversità, sul fatto di essere "inadatto ai tempi" (SS, 33) o "inadatto per questo mondo" (SS, 127). Essa risalta soprattutto in una Lettera al direttore apparsa su Il Mondo nel 1956, in cui Flaiano spiega il proprio "culto privato della Libertà":

Io dunque, limitandomi ad un culto privato della Libertà, non sono inserito nei miei tempi. Vorrei cavarmela, insomma, e salvare la faccia, amando la Libertà: impegno che non mi costa nulla, perché l'amiamo tutti ovviamente, anche se ognuno dandole un diverso scopo e significato. Per difendere questa Libertà che io dico di amare, io dovrò invece definirla, darle un programma, rifletterla nei miei scritti, farle dei proseliti. Ebbene, direi di no. (SS, 35)

La frase finale si avvicina per molti versi alla Filosofia del rifiuto, noto scritto critico raccolto nel postumo Diario degli errori (1976), in cui l'autore propone di "preferire sempre di no" (Scritti postumi, 374). Lidea di esclusione si trasforma frequentemente in un deciso rifiuto delle convenzioni (e delle convinzioni) letterarie, sociali e politiche correnti. Talvolta Flaiano asserisce pure di preferire il silenzio - ma si tratta, ovviamente, di un silenzio apparente, continuamente rotto dalla volontà di esprimersi come risposta radicale al cosiddetto "molto rumore per tutto" (Scritti postu$m i, 436)$ caratteristico della società contemporanea. L'explicit di questa lettera conferma quanto l'autore voglia presentarsi in veste di outsider, libero da qualsiasi costrizione: "dovrò prepararmi ad un'altra (ventennale?) relazione clandestina: una relazione disapprovata da tutti, in alto e in basso, a destra e a sinistra" (SS, 40). Anche negli articoli degli anni Settanta Flaiano ribadisce di appartenere alla "minoranza silenziosa" ("Sono di quei pochi che non hanno più nulla da dire e aspettano" [SS, 355]) o accenna alla propria situazione notando che "oggi [gli eremiti] preferiscono tornare nelle grandi città, dove vivono nella più perfetta delle solitudini, come lebbrosi. Infatti, non servono a niente." (SS, 343)

Oltre a queste prese di posizione esplicite, l'allontanamento di Flaiano si riflette anche nel modo in cui le 'situazioni' de La solitudine del satiro vengono narrate e focalizzate. In vari casi, la storia è narrata da un personaggio che non partecipa attivamente alle vicende, ma che osserva il mondo circostante in modo distaccato, non riuscendo a capire le smanie degli altri uomini. Questa incomprensione spicca già alla fine del primo racconto, in cui il narratore-protagonista dà un passaggio a un militare che si vanta delle proprie conquiste mostrandosi poco sensibile ai sentimenti femminili: "A M. [...] il lugubre dongiovanni scende, di nuovo stanco e pieno di disgusto, lasciandomi a riflettere sulle sue avventure." (SS, 11) In 
un altro racconto l'io narrante, capitato anzitempo in un cinema e costretto a guardare le pubblicità sullo schermo, esprime la sua incomprensione mediante due domande retoriche:

Quando cominciò il film vero e proprio mi sentivo non solo stanco ma turbato dall'idea di non essere nel mio tempo, di non amare la società, di "non conoscere i giovani". Quei giovani sullo schermo che enunciavano assiomi erano dunque tutto il mio prossimo? Possibile che non avessero altro da dirmi? (SS, 281)

In un altro caso il protagonista è invece un uomo sedentario e isolato dal mondo, che legge malvolentieri la posta e i giornali perché suppongono una comunicazione attiva: "Dopo i sogni tranquilli [...] della notte, il risveglio porta sul mio letto le sue minacciose simbologie, nei sogni più abbietti che la posta e i giornali mi suggeriscono." (SS, 27) Anche in altri racconti, l'atto della lettura — di lettere, giornali o libri - evidenzia la spaccatura tra l'io narrante e il mondo, presentandoci un lettore alienato, sbalordito o cinico. In queste occasioni, Flaiano ricorre inoltre volentieri all'ironia, inserendo dei commenti antifrastici ("Curiosa e gradevole lettura” $[S S, 99])$ che rivelano la distanza tra l'io e il mondo.

Altrove, il narratore è invece un osservatore distaccato che riferisce soltanto le cose che vede, senza interpretarle. È il caso del brano seguente, in cui si osserva (o si inventa?) il comportamento di alcuni bambini al mare:

Fregene. Un bambino sui sei anni che gioca ostinatamente ai bigliardini portandosi da casa uno sgabello, perché altrimenti non ci arriva. Un altro che compra la pistola ad acqua e la prova a lungo, senza ridere, e infine non ne sembra soddisfatto. Un ragazzetto benestante in vacanza, sui dodici anni, che gira sempre solo, lo sguardo serio e assorto. Ha già la sua motocicletta e non si muove che in motocicletta. (SS, 131)

Benché questa serie di frasi prevalentemente nominali non sia accompagnata da alcun intervento critico del narratore, essa denuncia nondimeno un aspetto preciso della contemporaneità, vale a dire l'inquietante precocità dei bambini, inestricabilmente legata agli ideali della società del consumo. Anche quando Flaiano 'trascrive' (inventa) un dialogo senza alcuno commento, come nel frammento "Vestirsi", in cui un'intera famiglia "non [sa] che mettersi" (SS, 372) per andare a una festa, critica ovviamente la superficialità della vita moderna. La mancanza di un'autorità narrativa non implica quindi che la critica sia meno vigorosa. Ciò si prova a fortiori quando la voce narrativa è pressoché assente, o si limita soltanto ad una 'constatazione' oggettiva: "Pena e sospetto che suscitano le persone normali in 
un mondo dove interessa soltanto l'Eccezionale, in tutte le sue varietà." (SS, 154 , il corsivo è mio)

Con questi esempi, che svelano l'arguzia narrativa con cui lo scrittore 'distaccato' esprime la sua critica della società contemporanea, non si vuole mettere in dubbio il profondo disagio vissuto da Flaiano durante gli anni del 'miracolo economico'. Alcuni articoli raccolti ne La solitudine del satiro, e soprattutto il diario "Fogli di via Veneto", rivelano infatti quanto fosse sincera la sua inquietudine. Il suo sentirsi 'inadatto al tempo' si rispecchia in modo particolare nella figura del poeta Cardarelli', esponente di un ideale letterario prebellico, che è tornato a Roma dopo la guerra sfidando "le regole del gioco, le quali vogliono che il vecchio elefante vada a nascondersi lontano, per morire" (SS, 246). Quando il narratore esamina il volto del rondista, non casualmente si richiama al topos del lago, luogo della contemplazione, specchio dell'anima nonché simbolo di uno "spietato scandaglio nella nostra memoria individuale e collettiva" e metafora di "una bellezza [...] sopravvissuta al disastro del moderno" (Benozzo, 250), per esprimere la comune malinconia:

Ero seduto al caffe e indugiavo a scrutare l'arrivo della notte nel volto e negli occhi di Cardarelli, come ci succede di scrutarlo in un lago, e me ne veniva una profonda malinconia, quasi che quel volto riflettesse il mio, e la mia giornata persa, resa più angosciosa dalla certezza di un'altra giornata e poi di un'altra, in questa via senza uscite. $(S S, 251)$

La posizione ai margini, che negli articoli si riflette in espedienti letterari di natura diversa, è quindi dettata da uno scoramento generale, che, secondo Longoni, nasce dal

constatare lo squallore di un popolo disorientato di fronte alla democrazia, che non si trova per nulla a suo agio nella riconquistata libertà, che all'impegno di una faticosa ricostruzione preferisce il piacere di un'evasione irresponsabile. ("Un piccolo maestro postumo", XXIX)

Ne La solitudine del satiro, Flaiano critica anzitutto il modus viven-

7 Interessante l'osservazione di Ruozzi riguardante la 'società del caffe': "A Flaiano piacciono gli scrittori e gli artisti che vogliono 'guastare la festa agli altri'. Gli piacciono i 'malpensanti' Leopardi, Barilli, Cardarelli, Longanesi, quelli che non hanno casa e sono inquieti ovunque, come lui; gli scrittori fuorimoda e contromoda, che ostentano la ricchezza della propria povertà anche esistenziale, appunto come Barilli e Cardarelli, vecchi e poveri ma ancora freschi di mente, capaci di irridere gli altri e se stessi." (Ruozzi, 23) 
di dell'italiano medio, che giudica 'volgare' o pure 'stupido', scagliandosi in particolare contro il sensazionalismo della cronaca, ritenuta responsabile di questa degradazione. Per questo la paragona per esempio a uno dei più famosi modelli letterari di 'immoralită8: "Come Lady Chatterley che, di un bosco, la cosa che più la colpiva era il guardaboschi, così la nostra cronaca vede, della vita, solo le passioni." (SS, 32) La comparazione è naturalmente dettata dall'impudicizia della protagonista del romanzo di D.H. Lawrence, ma sembra alludere anche alla differenza fondamentale tra la banalità della cronaca e i valori tradizionali della letteratura. In altri articoli si 'riproducono' invece dei dialoghi o dei monologhi assurdi, privi di ogni intervento critico, per denunciare la spettacolarizzazione della vita italiana moderna. Così, un'intervista inizia con la domanda "E dopo questo suo primo omicidio, che ha avuto tanto successo, che cosa ci sta preparando di bello?" (SS, 28), mentre un annuncio televisivo è riportato come segue:

"Signore e signori, la trasmissione è stata sospesa per ragioni tecniche. Tra qualche giorno, sopita l'ultima minaccia, riprenderemo i nostri giochi di società, che danno alla nostra vita nazionale quel senso di vivacità che tanto ci lusinga. Cercheremo di venire incontro ai più sciocchi, agli esibizionisti di ogni tendenza, terremo qualche processo piccante, pubblicheremo le vostre memorie, compreremo calciatori ungheresi. L'essenziale è arrivare con fiducia alla prossima estate; dopodiché, riaperti i concorsi di bellezza, i premi letterari, i festival e le danze, potremo affermare a fronte alta che all'estero ci invidiano la nostra allegria." (SS, 49-50)

Altrove, Flaiano prende di mira il turismo ("Il turista è un essere privilegiato, che non rimane ferito da ciò che vede" [SS, 212]), l'inquinamento ("I bagnanti romani, che condividono con il cuculo la tendenza a sporcare il proprio nido" [SS, 330]), l'urbanizzazione ("Anno nuovo, casa nuova" [SS, 187]), o la pubblicità ("Dove sono i santi? Dovremo accontentarci di morire in odore di pubblicità" $[S S, 93]$ ) sintomi vistosi di una malattia sociale incurabile9. Infine non sorprende che la partecipazione a una riduzione teatrale di Bouvard et Pécuchet inviti a criticare la stupidità

8 In questo articolo del 1956, Flaiano allude forse anche al fatto che Lady Chatterley's Lover fu pubblicato appunto in Italia (nel 1928) e vietato in Inghilterra a causa delle scene sessuali esplicite. Il romanzo di D.H. Lawrence apparirà sul mercato inglese soltanto nel 1960.

9 L'attualità degli scritti flaianei è stata notata da Sergiacomo: "Le frecciate del 'satiro solitario' si allargano così a raggiera toccando infiniti aspetti della vita italiana e tessendo una fitta rete concettuale, che dà al libro la veste di un inquietante e anche profetico documento della pervicace durata dei nostri problemi e della superficialità con cui sono stati nel tempo affrontati o elusi." (137) 
della società contemporanea ${ }^{10}$ :

[...] da allora la stupidità ha fatto progressi enormi. È un sole che non si può più guardare fissamente. Grazie ai mezzi di comunicazione, non è nemmeno più la stessa, si nutre di altri miti, si vende moltissimo, ha ridicolizzato il buon senso, spande il terrore attorno a sé. (SS, 310)

È chiaro che la stupidità fosse un bersaglio importante per Flaiano, che si richiama tanto a illustri precedenti letterari quali Flaubert ${ }^{11}$, quanto alla propria ironia ("Non saremo mai dei bravi, autentici, normali americani". $(S S, 127)$ La sua visione della società italiana del dopoguerra si riassume insomma bene nell'aforisma "Per vivere bene non bisogna essere eccessivamente contemporanei” (SS, 301), in cui spicca un'altra volta il ritratto di un autore emarginato, osservatore distaccato di un mondo in sfacelo.

Se Flaiano si distingue così con risolutezza dalla società volgare in cui vive, proclama anche, e con lo stesso vigore, la sua indipendenza rispetto ai colleghi letterati, essendo contrario tanto al modo in cui essi rappresentano la contemporaneità quanto al modo in cui assimilano i 'vizi' moderni. Di fronte al centro letterario, preferisce per definizione una posizione di rifiuto, un rifiuto che è sempre latente nei suoi articoli, o come attesta Manganelli, "un'eco lacunosa ma sempre intellegibile: la sillaba 'no".

Questo rifiuto, che riguarda il genere (in primis il romanzo), lo stile, l'impegno politico e l'ambiente letterario, è sempre dettato da un'avversione per la transitorietà delle mode culturali. In questo modo, Flaiano promuove un altro modello letterario, che ritiene insieme idoneo alla modernità e rispettoso dei valori tradizionali della letteratura.

10 Nel 1956 Moravia aveva già paragonato il Diario notturno flaianeo al romanzo incompiuto dello scrittore francese: "Effettivamente l'accanimento di cacciatore di farfalle con il quale Flaiano colleziona e incasella le assurdità, le sciocchezze, e le empietà dei suoi contemporanei rammenta l'analogo ossessivo atteggiamento flaubertiano." (105)

11 L'interesse per la stupidità di matrice flaubertiana è condiviso dall'amico Vitaliano Brancati, scomparso nel 1954 e ricordato da Flaiano nei "Fogli di via Veneto": "Poche sere fa lo incontro che usciva dal solito caffe, in fretta. Ci salutiamo appena, poi lui sente il bisogno di tornare indietro e dandomi la mano dice: 'Parto tra poco e non sono sicuro che ci rivedremo. Volevo salutarti.' Gli ho risposto poche parole, scherzando. Andava a Torino, per farsi operare. Adesso siamo qui allo scalo merci della stazione, davanti a un carro dove sopra c'è una bara. Ci siamo quasi tutti, gli amici della notte, le facce un po' sbiadite dallo scirocco." (SS, 258) Per un'analisi della raffigurazione 'postuma' del libro nelle opere brancatiane quale sintomo della stupidità contemporanea rinvio al contributo di Sabine Verhulst, "La noia di Adamo". 
Com'è stato rilevato dalla Corti ${ }^{12}$, Flaiano è sempre stato considerato uno scrittore irregolare anzitutto a causa della problematica assegnazione dei suoi 'frammenti' a un genere letterario specifico. In realtà, l'autore stesso ribadisce quest' idea di irregolarità nei suoi testi, riconoscendo a più riprese le difficoltà che incontra quando deve fornire una storia letteraria ben costruita. In uno dei primi articoli della raccolta il narratore-scrittore afferma per esempio di non riuscire a ideare una storia per il cinema:

Siamo in campagna, io e un amico, seduti davanti al cancello della sua casa. Dobbiamo parlare di una storia, per il cinema, e nessuno ha voglia di dire la prima parola, come se a scatenare le modeste immagini che suggeriscono appunto la storia e la sua protagonista, questo nostro pomeriggio perdesse ogni senso e il paesaggio scomparisse. (SS, 20)

L'incapacità di costruire una storia si trasforma poi nella denuncia dell'invenzione letteraria quale realtà falsa e inutile: "Un reciproco risentimento per la nostra futile pretesa di adattare la vita degli altri alle nostre necessità ci fa imbronciati." (SS, 22) L'osservazione rassegnata che chiude il racconto ("Infine, stanchi, decidemmo di riparlarne l'indomani. Non si inventa niente all'aperto. E copiarsi, che mal di testa!" [SS, 22]) contiene, infine, una critica al carattere uniforme delle finzioni letterarie con cui Flaiano sembra rivendicare la propria originalità e indipendenza.

La figura dello scrittore 'incapace' trova una variante evidente nello scrittore 'restio' o 'pigro'. Si pensi, per esempio, al narratore che descrive un dialogo divertente svoltosi in un ristorante romano per concludere in modo inaspettato: "Sono tornato a casa e ho scritto una lettera per rifiutare un certo lavoro. Adesso resta da vedere se la spedirò" (SS, 350); o a un altro brano in cui il protagonista decide di scrivere un saggio ma "alle 16, visto che niente succede, ci rinunzi $a$ " (SS, 103). In realtà, la negazione di una creazione letteraria omogenea, raffigurata alternativamente sotto la forma di inettitudine, pigrizia e renitenza, corrisponde semplicemente alla preferenza per un modello letterario alternativo, caratterizzato da un procedimento 'induttivo' che si rispecchia a sua volta nella forma breve degli scritti. Per riprendere le parole della Corti,

insoddisfatto della tradizionale routine narrativa, [Flaiano] per tutta la vita volgerà lo sguardo inquieto e cauto a una serie di particolari, di piccole cose, a ciò che magari è un nulla per gli altri, ma per che lui cresce e si organizza in un sistema mentale di lucida interpretazione della realtà. (XXVI)

12 Corti osserva che Flaiano "operava originali trasgressioni nell'uso dei generi letterari, bruciandone per lo più gli statuti in un'epoca come gli anni Cinquanta in cui l'istituzione letteraria dei generi funzionava ancora vivacemente" (X-XI). 
$\mathrm{Ne}$ La solitudine del satiro colpisce in particolare il rifiuto della forma romanzo, considerata inadatta a rappresentare il mondo contemporaneo: così, in un racconto che sfiora il surrealismo un personaggio riesce a scappare dal romanzo perché "lo scrittore pigro dormiva" (SS, 206), mentre il cosiddetto 'ritrattino' di un romanziere ci presenta un uomo incapace e nervoso che "aveva perso un po' di vista i suoi personaggi. Se ben ricordava, nell'ultimo capitolo li aveva lasciati a letto, ora non sapeva come riprenderli" (SS, 103). Benché un po' forzato, questo tipo di esperimento metanarrativo permette a Flaiano di manifestare il suo giudizio negativo sul romanzo, inteso come struttura sterile a causa della quale "l'ispirazione, mortificata, devia, intoppa, si esaurisce" (SS, 105). La critica sembra più efficace in un altro brano, con apertura palesemente ironica:

Certe volte mi sveglio nel cuore della notte, pieno di rimorsi, chiedendomi: "Che cosa sto facendo per il Romanzo?". Ora, mi interessa questa storia del romanzo come operazione critica, che cosa dev'essere un romanzo, come il narratore deve porsi davanti ai fatti. (SS, 340-341)

La stessa critica si ritrova in un'intervista (reale o inventata?) inclusa in un altro articolo, dove l'intervistato risponde all'accusa di scrivere troppo poco dicendo di non avere una vocazione narrativa: "Scrivo, che è una cosa molto diversa" (SS, 276). Il rifiuto di uno stile artificiale (o 'narrativo'), evidente in tutti gli esempi qui riportati, si rispecchia anche nei titoli originali di diversi articoli raccolti ne La solitudine del satiro, che ricordano appunto il 'diario' o il 'taccuino'13, suggerendo così fin dall'inizio una mancanza di struttura. Anche nei testi stessi l'autore insiste talvolta sul carattere disarticolato della propria scrittura, dichiarando per esempio di preferire una presentazione non cronologica dei fatti:

Queste note, scritte in vari momenti, non sono qui in ordine cronologico. Quello che volevo ricordare è una strada, un film, un vecchio poeta: cose disparate che si mescolano poco chiaramente non solo nella memoria ma anche in un diario. $(S S, 235)$

13 La solitudine del satiro contiene, tra l'altro, venticinque articoli apparsi nella rubrica "Diario notturno" su Il Mondo tra l'estate del 1956 e la primavera del 1960 (i pezzi anteriori furono raccolti da Bompiani nel libro omonimo) e dieci articoli della rubrica "Taccuino notturno", che apparve sul Corriere della Sera tra il 1969 e il 1972 (e di cui una parte fu già inclusa nel capitolo "Occasioni" de Le ombre bianche). Tutti gli articoli raccolti ne La solitudine del satiro sono riportati senza titolo. 
L'amalgama di "cose disparate che si mescolano poco chiaramente", caratteristico dei testi flaianei, ricorda non a caso la satura lanx, "piatto rituale, ricolmo di cibi diversi” (Brilli, 11) da cui è derivata la parola sati$r a$, con alcune connotazioni significative. L'origine etimologica della satira si rispecchia infatti tanto nelle "caratteristiche formali di questa pratica sans genre ai confini del letterario che predilige la mescolanza e la saturazione delle forme [...]" (11) e rifiuta la forma chiusa, quanto nella "valenza desublimante, decostruttiva" volta a "rappresentare la materialità eteroclita, polimorfa con tutte le sue contraddizioni, erigendola a specchio perturbante di ogni riduzione ed omogeneizzazione ideologica ed estetica" (12). Il legame tra aspetto formale e visione del mondo, peculiare della satira, è appunto l'alternativa letteraria offerta dal satiro Flaiano nella prima raccolta postuma.

Si nota quindi che la frantumazione 'satirica', tipicamente flaianea, non corrisponde in fondo all'incapacità di creare delle storie organiche (del resto, non si dimentichi che già nel 1947 l'autore aveva provato il contrario, vincendo il primo Premio Strega con il romanzo Tempo di uccidere). La disorganizzazione, che contraddistingue le sue opere più recenti, si rivela invece una vera e propria scelta critica con cui Flaiano vuole distanziarsi dai generi letterari correnti, non senza lanciare invettive contro l'artificiosità di molta letteratura contemporanea.

Accanto alla frantumazione del testo letterario in piccole unità autonome, Flaiano vuole anche distinguersi dal letterato 'medio' con uno stile e una lingua notevolmente piani. Come ha precisato Siciliano, la cura con cui egli ha limato i suoi scritti rispondeva al desiderio di "depurare la lingua viva dalla sua connaturata gergalità senza tradirla." (176) Ne La solitudine del satiro lo stile elementare emerge sin dal primo racconto ("Un militare chiede un passaggio" [SS, 9]), costituito quasi interamente da frasi che seguono lo schema standard Soggetto + Verbo + Oggetto, con un conseguente ritorno dell'indicativo presente. Talvolta, un frammento può contenere una sola frase: per esempio "Riceve i ladri cattolici e tiene loro un discorsetto sul modo di rubare il necessario ai poveri, che hanno bisogno solo del superfluo, altrimenti è la rivoluzione" (SS, 16-17), che mantiene una struttura grammaticale elementare nonostante la paradossalità dell'affermazione. Anche gli aforismi combinano comunemente un contenuto paradossale con una notevole chiarezza formale: "Oggi il cretino è pieno di idee." (SS, 311)

Che in questo modo lo scrittore volesse distanziarsi dalla raffinatezza del linguaggio letterario, preferito da molti dei suoi colleghi, risulta chiaro da un articolo del 1956, dove compaiono due brani estratti da un fittizio 
diario di un letterato, il cui stile contrasta diametralmente con quello dei diari flaianei:

L'estate trionfa qui coi suoi modi usuali e sempre sorprendenti, con una forza così languida che mi ha spinto a considerare la vita di tutti nella sua essenza, a farmene artefice. Ho scritto "artefice". Questa parola, dopo l'uso smodato fattone da poeti a me inferiori, può apparire sospetta: ma io amo questo e altri simili vocaboli, che sembrano attrezzi di ferro battuto, per quel tanto di civetteria desueta che imprimono alla mia prosa, per altri versi perfetta. $(S S, 52)$

Anche in un altro brano, che contiene una breve constatazione: "Poeta e contadino, ieri. Oggi, prosatore e agrario, saggista e coltivatore diretto, filosofo e datore di lavoro" (SS, 146), è implicita la critica della complessità linguistica. Qui si vede che lo scrittore non si scontra soltanto con la lingua letteraria corrente, ma anche con i termini astratti caratteristici del giornalismo. La critica del gergo giornalistico è chiara nel divertente "ritrattino" di un cronista che teme di aver contratto "un epatema (?)" (SS, 106), ma che alla fine deve costatare che il suo malessere non è appunto fisico: “'Dio mio', pensò, 'eppure ero così bravo in italiano!'” (SS, 107) o in questa imitazione ironica del linguaggio giornalistico, presentato come rigido, convenzionale e prevedibile:

Nei commenti politici, la situazione è sempre oscura, la svolta sempre pericolosa, il chiarimento sempre necessario, l'accordo sempre augurabile, l'alta parola del Pontefice suona sempre monito, la distensione non deve far diminuire la vigilanza e gli esempi vanno presi dalla storia recente. $(S S, 198)$

Tanto nella letteratura quanto nel giornalismo (e infine nella burocrazia $\left.^{14}\right)$, Flaiano condanna quindi il ricorso a termini rari o oscuri, argomentando che essi non possono avere un rapporto trasparente con la realtà circostante. In un articolo più recente, del 1970, egli spiega la sua concezione dello 'scrivere chiaro', reagendo al parere negativo di un amico su un suo libro:

Quel che un amico mi rimprovera, con dolcezza e anche simpatia, è che il dettato sia chiaro. Si capisce tutto. "Non devi aver faticato molto" mi

14 "Cosicché, volta a volta, noi siamo astanti, iscritti, sottoscritti, presenti, passanti, contribuenti, utenti, usufruttuari, richiamati, abbonati, associati, ricorrenti, fedeli, credenti. Siamo destinatari, mittenti, depositari, conducenti, correntisti, coniugi, parenti, acquirenti. Siamo in carica, in ausiliaria, in aspettativa, in ferie, fuori posto. Brilliamo dunque sempre sotto aspetti diversi ma per motivi precisi, mai per quel che crediamo di essere in sostanza. E quando lo crediamo, allora eccoci imputati." (SS, 102) 
dice con indulgenza. Rispondo che, al contrario, ho faticato moltissimo, che ho scritto e riscritto pagine infinite volte, poiché se avessi dato ascolto alla mia natura tutto sarebbe rimasto nel vago e nell'oscuro. "Non ami gli esperimenti', insiste l'altro. (SS, 321)

Successivamente, Flaiano difenderà il suo modo di scrivere in un ragionamento che si conclude appunto con l'affermazione perentoria: "noi, la vera operazione sperimentale la facciamo scrivendo "chiaramente" (SS, 323). In tal modo le mode neo-sperimentali degli anni Sessanta - di cui l'anonimo amico sembra essere un seguace - vengono interpretate come sostituti artificiali e scadenti della vocazione letteraria: "Mi fa sorridere lo scrittore che esaspera la punteggiatura, la scelta dei tempi e dei modi, la metonimia e la metafora, o pesca vocaboli obliati e li usa in un'accezione squisita." (SS, 323)

Presentandosi come escluso - restio, accidioso o biasimato - Flaiano promuove quindi una letteratura indiscutibilmente atipica nel panorama italiano del secondo dopoguerra, caratterizzata da una frantumazione strutturale e da un'elementarità stilistica. Tuttavia, il distanziamento radicale che si riscontra ne La solitudine del satiro sembra anche - e forse soprattutto - dettato da una sensazione di disagio nei confronti dell'impegno politico di molti letterati, cineasti e artisti contemporanei. Se il rifiuto flaianeo dell'engagement, inteso come obbedienza a una linea politica precisa, nel clima neorealista del dopoguerra ${ }^{15}$ e negli anni precedenti i 'fatti di Ungheria' risulta molto reciso, la sua critica nei confronti della politica non scema dopo il 1956, protraendosi fino all'indomani delle contestazioni studentesche. I suoi articoli pungenti non colpiscono dunque soltanto il "fronte della sinistra" (Brunetta, 128) e l'intellettuale organico di stampo gramsciano, ma anche i diversi governi democristiani, offrendo così un'immagine del tutto negativa della politica italiana nell'era repubblicana.

In La solitudine del satiro, come già nel Diario notturno, Flaiano insiste sul fatto di essere apolitico, e di conseguenza, 'poco' italiano. Così, in un articolo del gennaio 1957, elabora una risposta polemica alla domanda di "un tale" (giornalista) che gli telefona per sapere di quale nazionalità vor-

15 Emblematica, a questo proposito, la recensione flaianea di La terra trema di Visconti apparsa su Il Mondo il 17 gennaio 1950, in cui il film neorealista viene descritto come "una riprova della nota verità che gli argomenti politici possono arricchire un'opera d'arte soltanto se assorbiti e trasformati poeticamente, senza di che si resta nella polemica, nel pamphlet o in quell'arte di partito che chiede agli iscritti più rinuncia e abnegazione che giudizio critico." (Lettere d'amore al cinema, 172) 
rebbe essere se non fosse italiano16: "Prima di tutto bisognerebbe provare che sono italiano. [...] non sono fascista, non sono comunista, non sono democristiano: ecco che mi restano forse venti probabilità su cento di essere italiano." (SS, 74-75) In un altro articolo si legge invece che egli "non salut $a$ né stringe le mani agli enti, alle associazioni, alle mafie, ai dogmi, alle ragion di stato" (SS, 46), statement provocatorio che ricorda appunto il nesso tra 'culto privato della Libertà' e 'filosofia del rifiuto'. In altre occasioni, l'autore allude più sottilmente alla propria apoliticità: quando il protagonista di un racconto si reca al caffe Rosati, osserva per esempio che " $\mathrm{Ci}$ sono tutti i miei amici, e uomini politici” (SS, 142), frase in cui la virgola superflua produce una distinzione netta tra 'noi' e 'gli altri'; quando l'autore nella "Lettera al direttore" passa in rassegna argomenti "dal più facile al più capzioso, dal più onesto al più politico" $(S S, 35)$, discredita la parola 'politico' tramite una falsa contrapposizione (con 'onesto') e un parallelismo sottile (con 'capzioso').

$\mathrm{Ne}$ La solitudine del satiro Flaiano si schiera frequentemente contro le direttive impartite dal governo democristiano negli anni del dopoguerra. Un articolo del marzo 1959, per esempio, è interamente dedicato al 'resoconto' della situazione in cui si potrebbe trovare il paese nel 1965. Questi appunti di un italiano anonimo tornato nella "bella Italia" $(S S, 183)$ traboccano d'ironia: "Quanto all'assistenza sanitaria dev'essere ottima, visto che non si costruiscono più ospedali [...] ma soltanto stadi sportivi e cinematografi" (SS, 187), mentre "una nuova legge, opportuna, proibisce l'uso di materiali molto solidi, sicché è scongiurato il pericolo di ogni futura crisi edilizia" (SS, 187). Se l'ironia si trasforma però in sarcasmo, emerge anche la profonda delusione di Flaiano nei confronti di un establishment politico che manca di coraggio: "Che fortuna essere nati in un paese che ritarda la soluzione dei suoi grandi problemi con le piccole mance. Che antica saggezza." (SS, 192)

Benché la delusione politica di Flaiano provenga in buona parte dalla linea governativa della DC, l'autore attacca ancora con maggior vigore la "chiesa" comunista ${ }^{17}$. Il PCI è considerato "un partito conservatore e reazionario, che non vuol fare rivoluzioni e si accontenta che gli altri partiti lo credano capace di farle" (SS, 38), mentre i suoi membri hanno la smania di "negare il Sistema senza muovere un passo per uscirne" (SS, 316). In un

\footnotetext{
16 Una simile inchiesta era apparsa su Epoca il 20 febbraio 1955 con il titolo "Se non foste italiano che vorreste essere francese, turco, tedesco, armeno?", domanda cui risposero fra l'altro De Chirico, Visconti, Ungaretti, Buzzati e Parise.

17 "Il comunismo è una chiesa che prospera nel martirio." (SS, 57)
} 
articolo, apparso il 20 novembre 1956, l'autore si scaglia perfino violentemente contro la posizione presa dai comunisti italiani - tanto da un "giovane amico comunista" (SS, 46) quanto da Togliatti o dai giornalisti de L'Unità - dopo la rivoluzione ungherese, rimproverando loro viltà e mancanza di raziocinio ("I carri armati russi hanno eliminato ogni dubbio nelle loro menti" [SS, 46-47]). Flaiano non critica soltanto il carattere pseudorivoluzionario del marxismo di marca italiana, ma si infastidisce anche per l'insensata popolarità del PCI. In un racconto intitolato appropriatamente "L'uovo di Marx"18, i protagonisti discutono perciò sul modo in cui combattere l'ideologia di sinistra, concludendo che si dovrebbe introdurre "l'insegnamento obbligatorio di comunismo nelle scuole medie e universitarie" $(S S, 60)$ affinché gli studenti comincino a odiare il marxismo: "non perché l'ignorano - come in molti casi succede oggi — ma perché sono obbligati a studiarci sopra" (SS, 61).

L'incomprensione politica, che deriva tanto dalla rigidità dei partiti quanto dal loro successo immeritato, è un altro motivo per cui Flaiano si sente escluso dal campo letterario, in quegli anni appunto dominato dalla questione dell'engagement. Ne La solitudine del satiro l'autore ribadisce di frequente il proprio disinteresse per la politica, ricorrendo, per esempio, a un narratore — di nuovo scopertamente autobiografico - che, sfogliando un giornale, comincia a fantasticare sulla notizia di un assalto di topi a Napoli invece di commentare un articolo su Sartre ${ }^{19}$ sulla pagina precedente: "E triste scherzare su questi argomenti, ma non me la sento di attaccare Sartre” (SS, 194). Specie durante la rivolta ungherese, Flaiano denuncia l'opportunismo e l'ipocrisia degli intellettuali impegnati, 'scultori' di idolatrie transitorie:

Sguazziamo nel disgelo. Le statue di neve di Stalin, con la pipa in bocca e la scopa sottobraccio, si squagliano al primo sole. Gli scultori, che imponevano ai cittadini di adorarle, ora si tirano palle di neve sporca tra di loro, aumentandone il peso con un sasso. Uno di essi provoca un passante: vuole il dialogo. $(S S, 33)$

In un altro articolo si descrive la condotta ipocrita di "un eroe del nostro tempo", che nel '43 "si toglieva il distintivo alle due del pomeriggio"

18 "È un'idea talmente semplice che mi meraviglio non sia venuta a nessuno. $\grave{E}$ l'uovo di Marx." (SS, 58)

19 In un altro articolo Flaiano osserva che Sartre "passa l'esistenza a entrare e a uscire dal partito comunista", paragonandolo a "l'ometto con l'ombrello di certi barometri che indicano così il buono e il cattivo tempo" o al "cane dell'ortolano, che detesta i cavoli ma pretende che nessuno li tocchi” $(S S, 48)$. 
(vale a dire l'8 settembre) mentre nel '49 elogia "un brutto film come espressione d'arte popolare progressista" (SS, 50). Il narratore elenca gli incontri con l'eroe' in modo cronologico (dal ' 45 fino a "l'altro ieri"), senza aggiungere un commento critico, ma lasciando tuttavia intravedere la sua incomprensione. Altrove, prima di riferire due brani dal diario di un letterato in cui parodierà appunto l'invadenza degli scrittori nella politica, il narratore avvisa il lettore che i fogli

smentiscono una favola corrente; e cioè che la letteratura sia staccata dalla vita politica e che i letterati, chiusi nelle loro torri d'avorio, misconoscono e deridono coloro che governano le sorti del Paese, né porgono loro alcun aiuto. (SS, 52)

Flaiano, 'antieroe' per eccellenza, sembra invece rinchiudersi in una torre d'avorio sui generis, luogo apolitico - o meglio apartitico - da cui poter criticare la presunzione politica dei suoi colleghi che si conformano volentieri alle ideologie in voga.

L'esclusione di Flaiano si traduce letteralmente in una opposizione alla socievolezza dei suoi colleghi letterati, che a suo parere rischiano di trasformarsi in una folla indistinta e acritica, dedita al semplice divertimento. L'autore-narratore è sorpreso in primo luogo dalla quantità di scrittori e artisti nell'Italia del boom economico, conseguenza del cosiddetto 'miracolo culturale': "Dipingono tutti. Il guaio è che dipingono male" (SS, 176); "Tutti scrivono. Chi non scrive sta raccogliendo il materiale. Se il miracolo culturale va avanti, avremo uno scrittore ogni cento abitanti." (SS, 248) Le annotazioni, che contengono ovviamente una buona dose di ironia, sottintendono nondimeno una profonda inquietudine nei confronti di una crescente comunità di artisti che mancano di una vocazione autentica, attratti come sono da una vita che è 'per forza' allegra. Infatti - e si noti l'ossimoro - "tutti si divertono accanitamente, nessuno si annoia" (SS, 186, il corsivo è mio), a eccezione di chi scrive: di conseguenza, la noia flaianea va interpretata come una reazione radicale alla frenesia della società contemporanea.

In vari articoli Flaiano passa in rassegna "le virtù" (i vizi) dei letterati romani, tra cui spicca anzitutto un "sano esibizionismo" (SS, 267), sintagma che accentua ovviamente l'eccessività di una tale tendenza. La volontà di mostrarsi, malattia di cui 'soffrono' molti dei suoi colleghi, colpisce soprattutto durante le consegne dei premi letterari, trasformate in ridicoli spettacoli commerciali ("Quest'anno, ogni scrittore ha avuto due premi e mezzo" [SS, 188]), nonché in occasione di altri momenti pubblici come il convegno sui rapporti tra Cinema e Letteratura tenuto a Roma nel 1957. 
Quest'ultimo dà luogo a una critica severa dello 'scrittore medio italiano', descritto come un individuo insieme presuntuoso e opportunistico:

C'è poi la tendenza dello scrittore medio italiano a considerarsi inviolabile come un sacerdote. Vuol dire messa ai cannibali, ma detesta il martirio; vuol convertire i suoi lettori, ma si preoccupa della pensione e delle percentuali; vuole la Libertà ma ammira anche in cuor suo le enormi tirature di Stato delle Repubbliche Sovietiche. (SS, 151)

La presunzione degli scrittori è un tema costante ne La solitudine del satiro: in un altro brano, ci si lamenta al plurale (ma si tratta naturalmente di un 'noi' falso) che "lo Stato dovrebbe aiutarci, ma lasciarci liberi di fare le nostre infantili sciocchezze" (SS, 141), o si decreta che l'autore engagé, convinto di essere un viaggiatore, è invece "un trasportato, un utente della ferrovia di massa, anche se beneficierà di notevoli riguardi e riduzioni" (SS, 342).

Particolarmente interessante a questo riguardo è la reazione di Giovanni Battista Angioletti al brano sopraccitato, pubblicata su Il Mondo il 3 dicembre 195720 . In questa lettera Angioletti, allora presidente del Sindacato Nazionale Scrittori, s'indigna per le "ingiuste cattiverie scritte da Ennio Flaiano" e implora pietà per gli scrittori che vivono "in un Paese che senza un fremito li lascerebbe morire di fame”. Flaiano risponde:

Voglio solo precisare che la mia nota andava vista alla luce dell'affetto che ho per gli scrittori in genere, tra i quali mi onoro di avere molti amici: e tenendo conto che anch'io spero di diventare, un giorno, scrittore.

La risposta di Flaiano è emblematica, non solo perché incompatibile con la dura critica espressa precedentemente, ma anche perché l'autore ci esplicita la sua autoemarginazione dal campo letterario. En passant, Flaiano denuncia la superbia dei letterati ("mi sorprende che gli scrittori, giudici preziosi della società, rifiutino ogni giudizio che li riguardi”), nonché la mancanza di umorismo del suo corrispondente e, per estensione, degli scrittori a cui si era rivolto prima: "ho esagerato, ma l'iperbole era chiara".

Escluso dal campo letterario, neanche 'scrittore', non sorprende quindi che Flaiano si scontri con gli 'idoli' letterari - e questa volta chiamati in causa con il loro nome - nel diario "Fogli di via Veneto": il narratore sogna di imbattersi, durante una passeggiata notturna, in un corteo rumoroso di scrittori, tra cui scorge, non a caso, il fondatore del premio Strega: "Bassani sottobraccio a Moravia, Goffredo Bellonci tra Alberto Arbasino e

20 Per quanto ne sappiamo, le lettere di Angioletti e Flaiano sono inedite. 
Italo Calvino: cantavano. Mario Soldati imitava se stesso" (SS, 271). Dopo l'arrivo di una massa entusiasta che applaude "senza sapere che cosa e chi" (SS, 272), il narratore, apparentemente allarmato, si chiede: "Non vedevo Pasolini. Dov'era Pasolini? Che la sua ritrosia l'avesse trattenuto a casa?" $(S S, 272)$ Con la dovuta ironia, il diarista descrive così un 'corteo' di letterati a cui non vuole appartenere, con cui non vuole identificarsi perché portatori di ideali - letterari, politici o sociali - che disapprova.

Il 'no' di Flaiano è quindi un rifiuto del hic et nunc in favore di una concezione meno contingente del mondo, al di là degli impulsi immediati che sono offerti tanto dalla società circostante quanto dalle mode letterarie. Ne La solitudine del satiro l'autore 'restio' esprime così la sua delusione nei confronti dei suoi colleghi che reagiscono volentieri a tali stimoli, negando il ruolo decisivo che può e che deve avere la letteratura all'interno di una società in decadenza. Contemplando i letterati della sua generazione che sono ancora, idealmente, "il sale della terra" - non tanto profeti che 'danno senso' quanto voci critiche che interrogano la società Flaiano si chiede sinceramente: "se il sale diventa sciapo, con che lo saleremo?"21 Paradossalmente, è stato il rifiuto dell'attualità a garantire il successo postumo e attuale a uno scrittore "dimenticato dalla storia"22.

\section{Universiteit Gent}

\section{OPERE CITATE}

Angioletti, Giovanni Battista. "Lettera". Il Mondo 3 dicembre 1957: 10.

Benozzo, Francesco. "Lago." Luoghi della letteratura italiana. A cura di Gian Mario Anselmi e Gino Ruozzi. Milano: Mondadori, 2003. 243-252.

Brilli, Attilio. La satira. Storia, tecniche e ideologie della rappresentazione. Bari: Dedalo, 1979.

Brunetta, Gian Piero. Il cinema neorealista. Storia economica, politica e culturale. Bari: Laterza, 2009.

Cattaneo, Giulio. "La solitudine del satiro." La critica e Flaiano. A cura di Lucilla Sergiacomo. Pescara: Ediars, 1992. 143-144.

Celenza, Franco. Le opere e i giorni di Ennio Flaiano. Ritratto d'autore. Milano: Bevivino, 2007.

Corti, Maria. Introduzione. Opere. Scritti postumi. Di Ennio Flaiano. Milano: Bompiani, 1988. VII-XLIII.

21 La citazione chiude la lettera a Angioletti.

22 In un'intervista con Giulio Villa Santa del 1972, Flaiano disse: "È probabile che io sia un antico romano che sta qui ancora, dimenticato dalla storia, a scrivere cose che altri hanno scritto meglio di me: [...] Catullo, Marziale, Giovenale." 
D’Angelo, Giacomo. Flaiano e D’Annunzio. L'Antitaliano e l'Arcitaliano. Chieti: Solfanelli, 2010.

Flaiano, Ennio. La solitudine del satiro. Milano: Adelphi, 1996. "Lettera". Il Mondo 3 dicembre 1957: 10.

—. Lettere d'amore al cinema. A cura di Cristina Bragaglia. Milano: Rizzoli, 1978. Opere scelte. A cura di Anna Longoni. Milano: Adelphi, 2010.

- Opere. Scritti postumi. A cura di Maria Corti e Anna Longoni. Milano: Bompiani, 1988.

- Opere. 1947-1972, a cura di Maria Corti e Anna Longoni, Milano: Bompiani, 1990.

Giammattei, Emma. "Flaiano." Solitudine del moralista. Alvaro e Flaiano. Di Antonio Palermo e Emma Giammattei. Napoli: Liguori, 1986. 117-190.

Longoni, Anna. "Fortuna critica." Opere. 1847-1972. Di Ennio Flaiano. Milano: Bompiani, 1990. 1467-1487.

—. "Nota alla presente edizione." Opere scelte. Di Ennio Flaiano. Milano: Adelphi, 2010. LXIII-LXV.

—. "Note ai testi." Opere. Scritti postumi. Di Ennio Flaiano. Milano: Bompiani, 1988. 1243-1353.

"Un piccolo maestro postumo." Opere scelte. Di Ennio Flaiano, Milano: Adelphi, 2010. VII-XXXII.

Maingueneau, Dominique. Le discours littéraire. Paratopie et scène d'énonciation. Parigi: Colin, 2004.

Maingueneau, Dominique e Patrick Charaudeau. Dictionnaire d'analyse du discours. Parigi: Seuil, 2002.

Manganelli, Giorgio. "Il segreto di Flaiano." Tuttolibri, 11 dic. 1976.

Marabini, Claudio. Introduzione. La solitudine del satiro. Di Ennio Flaiano. Milano: Rizzoli, 1989. I-VIII.

Milano, Paolo. "Amo la solitudine la satira e la noia." La critica e Flaiano. A cura di Lucilla Sergiacomo. Pescara: Ediars, 1992. 148-150.

Moravia, Alberto. "Lottimista di umor nero." La critica e Flaiano. A cura di Lucilla Sergiacomo. Pescara: Ediars, 1992. 104-106.

Pampaloni, Geno. "Flaiano: L'uomo e l'opera." Ennio Flaiano. L'uomo e l'opera. Atti del Convegno Nazionale nel decennale della morte dello scrittore. A cura dell'Associazione Culturale Flaiano. Pescara: Fabiani, 1989. 11-21.

Ritrovato, Salvatore, "Ennio Flaiano, i 'diari' di un anti-italiano." Carte urbinati 2009, n. 1: 179-184.

Roda, Enrico. "Così è se vi pare. Flaiano o la libertà.” Intervista con Ennio Flaiano. Radiocorriere gennaio 1962: 18.

Rosati, Gianni. "L'italiano non ride." Intervista con Ennio Flaiano. Opere. Scritti postumi. Di Ennio Flaiano. Milano: Bompiani, 1988. 1199-1208.

Ruozzi, Gino. Ennio Flaiano, una verità personale. Roma: Carocci, 2012.

Sergiacomo, Lucilla. Invito alla lettura di Ennio Flaiano. Milano: Mursia, 1996.

Schembri, Pascal. Un marziano in Italia. Vita di Ennio Flaiano. Villorba: Anordest, 2010.

Siciliano, Enzo. “Flaiano 'antropologo' degli anni Cinquanta." La critica e Flaiano. 
A cura di Lucilla Sergiacomo. Pescara: Ediars, 1992. 175-177.

Trubiano, Marisa S. Ennio Flaiano and His Italy. Postcards from a Changing World. Madison: Fairleigh Dickinson University Press, 2010.

Verhulst, Sabine. "La noia di Adamo. Immagini postume del libro in Vitaliano Brancati." Lettere italiane 2012, n. 3: 301-321.

Villa Santa, Giulio. "La satira, la noia e la fede." Intervista con Ennio Flaiano. Opere. Scritti postumi. Di Ennio Flaiano. Milano: Bompiani, 1988. 12231237. 